\title{
PARTIAL LAPAROSCOPIC ADRENALECTOMY - ANATOMICAL BASIS AND OPERATION TECHNIQUE
}

DOI: 10.36740/WLek202009215

\author{
Sergiy P. Styopushkin, Viktor P. Chaikovskyi, Volodymyr A. Chernylovskyi, Ruslan V. Sokolenko \\ CITY CLINICAL HOSPITAL № 4, DNIPRO, UKRAINE
}

\begin{abstract}
The aim: To optimize the indications for partial laparoscopic adrenalectomy (PLA), to give a detailed outline of a PLA technique and to provide technical tips to ensure safe and highly-effectiveness, based on the knowledge of adrenal anatomy and blood supply.

Materials and methods: Between January 2010 and September 2018, our department performed 47 adrenal glands surgeries. The operations included 29 total laparoscopic adrenalectomies (TLA), 4 open adrenalectomies (OA) and 14 partial laparoscopic adrenalectomies (PLA).

Results: The histopathological examination of all operated patients detected 9 (19.1\%) malignant tumors, including 5 metastatic tumors. Benign tumors includes 24 (63\%) adenomas, $8(21 \%)$ pheochromocytomas, 4 (10,5\%) cysts and 2 (5,5\%) ganglioneuromas. Post-PLA histopathological findings revealed 6 adenomas, 2 pheochromocytomas, 4 cysts and 2 ganglioneuromas.

Conclusions: Keeping in mind anatomical features of adrenal gland blood supply in highly-selected patients, PLA can be performed in a number of patients. 2 mm of a minimal resection margin is enough to preserve a false tumor recurrence. Anatomically grounded PLA is becoming a new standard of benign adrenal gland tumors treatment, providing an opportunity to save more adrenal gland functional tissue and to prevent hipocorticism development in postoperative period.
\end{abstract}

KEY WORDS: ganglioneuroma, pheochromocytoma, adrenal gland, partial laparoscopic adrenalectomy, adrenal gland adenoma

Wiad Lek. 2020;73(9 p. II):1977-1981

\section{INTRODUCTION}

The main goal of this article is to optimize the indications for partial laparoscopic adrenalectomy (PLA), to give a detailed outline of a PLA technique and to provide technical tips to ensure safe and highly-effectiveness, based on the knowledge of adrenal anatomy and blood supply. In this paper, a special focus is placed on the surgical technique of PLA. This operation was standardized and can be performed with good results.

In recent years, total laparoscopic adrenalectomy (TLA) substituted open adrenalectomy (OA) and became a gold standard for treatment of adrenal gland benign tumors.

The latest findings confirm the use of minimally invasive surgery for treatment of adrenal tumors and prove TLA to be an effective and safe method comparable to OA in operation time and complications rate, yet having the potential for shorter hospital stay, lower blood loss and better cosmetic effect.

PLA is a new step of treatment of adrenal tumors. The main advantage of this operation is a preservation of functional tissue of adrenal gland, which reliably helps to avoid hipocorticism. Walz [1] first provided minimal invasive partial adrenalectomy with retroperitoneal approach, and this surgery showed good treatment results and helped to avo rmone-replacement therapy. Janetschek described PLA-experience in patients with aldosterone-productive adenoma [2] and pheochromocyto- ma [3]. In the case of bilateral hormone-productive tumors (pheochromocytoma, aldosterone- and cortisol-productive adenomas), special emphasis is put on the advantages of this surgery, which makes it possible to preserve a bigger volume of functional tissue and reduce the risks of cortical insufficiency development in the case of contralateral tumor [4]. Knežević $\mathrm{N}$ [5] determined operative indications for tumors located predominantly in the adrenal gland margin. In his systematic review, Nagaraja [6] showed a recurrence free rate $8 \%(95 \%$ CI: $0.05 \mathrm{e} 0.12)$, whereby $85 \%$ of operated patients $(95 \% \mathrm{CI}$ : $0.78 \mathrm{e} 0.9$ ) did not require further post-PLA corticosteroid replacement therapy.

At the same time, despite the preservation of adrenal gland tissue or providing adequate steroid therapy, Addison crisis can be detected in $10-35 \%$ of patients [4]

Rich blood supply is a necessary condition for optimal adrenal gland functioning. Adrenal gland blood supply (Figure 1) is implemented 20-30 thin arterial branches from right superior adrenal aa. (from inferior phrenic a.), middle adrenal a. (from abdominal aorta) and inferior adrenal a. (from renal a.).

Sinusoid blood capillaries form central vein inflows into inferior vena cava near the right adrenal gland and into the right adrenal gland on the left. Adrenal glands (especially the left one) contain small veins, which flow into portal vein inflows [7]. 
The indications for organ-sparing adrenal gland operations include cystic lesions, benign tumors up to $4 \mathrm{~cm}$., especially hormone-productive tumors (e.g. aldosteronoma, glucocorticoid-, androgen- and estrogen-productive adenomas) and bilateral tumor. To absolute contraindications belong adrenal gland malignant tumors and metastatic tumors. Relative contraindications include extensive benign tumor mass, presence of multifocal tumors and poor blood supply to residual adrenal gland tissue. Sufficient amount of residual tissue is not yet determined. E.K. Diner [8] believes that the preservation of 3-5 $\mathrm{mm}$ is enough to achieve good postoperative results.

General contraindications include Cushing's disease (secondary hypercortisolism), severe cardiac pathology, heart failure, uncontrolled coagulopathy etc.

All patients underwent Abdominal and Retroperitoneal Ultrasound and contrast-enhanced (Tomohexol/Omnipack) Chest, Abdomen and Pelvis CT-scan. An endocrinologist and GP examined patients. Serum level of aldosterone, cortisol, catecholamines and urine metanephrine was measured. To exclude secondary hypercorticoidism (Cushing's disease), the patients with elevated adrenal gland hormones level underwent a high-dose overnight dexamethasone suppression test (DST).

Blood pressure was thoroughly measured before the surgery and intraoperatively to control hemodynamics in the time of operation and especially during adrenal gland manipulation.

\section{THE AIM}

To optimize the indications for partial laparoscopic adrenalectomy (PLA), to give a detailed outline of a PLA technique and to provide technical tips to ensure safe and highly-effectiveness, based on the knowledge of adrenal anatomy and blood supply.

\section{MATERIALS AND METHODS}

Between January 2010 and September 2018, our department performed 47 adrenal glands surgeries. The operations included 29 total laparoscopic adrenalectomies (TLA), 4 open adrenalectomies (OA) and 14 partial laparoscopic adrenalectomies (PLA). The surgeries on the right side made 27 (57\%) case, while those on the left side made $20(43 \%)$ ones. The median age of the patients was $63 \pm 12$ years, and their gender breakdown was $16(34 \%)$ male and $31(66 \%)$ female. The indications for OA included primary malignant tumors exceeding $6 \mathrm{~cm}$ as well as retrocaval location of the tumor. The PLA was performed for benign tumors from 1.5 to $4 \mathrm{~cm}$ large and for cysts with size up to $8 \mathrm{~cm}$. The left-sided PLA was performed in $8(57 \%)$ cases, and right sided - in $6(43 \%)$ cases. Technically, it was possible to perform the left-sided PLA in a bigger number of patients. It can be explained by a different location of the major left adrenal vein and a more stretched adrenal gland.

\section{RESULTS AND DISCUSSION}

As a rule, the right-sided PLA is more difficult to perform due to a shorter major adrenal vein. However, we discovered that the presence of additional veins to right adrenal gland enabled us to save more adrenal gland tissue with good blood supply, which in our investigation had been detected in 25\% patients (Figure 2). The PLA was performed with "cold" scissors following the preliminary clipping of resection border. The clipping was performed with large Hem-o-lok clips. N. Roukounakis [10] suggest reaching 3-5 mm of resection border. A traditional way of adrenal gland resection with electrocoagulation ligation has a serious disadvantage, lying in coagulation necrosis in resection margin, which reduces the volume of the adrenal gland functional tissue.

The patient is placed in lateral position at an angle of $60^{\circ}$; a soft roll may be placed under rib XI. 3-4 ports are used for the left-sided operation and 4 - for right-sided operation, including liver retractor. This operation employs 30-degree optical equipment. Following the pneumoperitoneum creation with the help of Veress needle, an 11-mm port is placed in the gap between costal arch and lateral margin of abdominal rectus muscle. It is also possible to perform the first port placement according to Hasson technique [11], which is especially beneficial in the case of repeated abdominal cavity surgery. Further, a 30-degree laparoscope is inserted and primary visual investigation of 4 abdomen quadrants is performed. After that, all ports are placed under visual control. Under direct visual control, the $11-\mathrm{mm}$ port for liver retractor is placed on linea axillaris media. The 11$\mathrm{mm}$ port is placed on linea medioclavicularis in infracostal region. Finally, following the liver retractor installation and retraction of the liver, the most medial 11-mm port is placed on linea parasternalis. To operate on left adrenal gland, it is possible to use a $6-\mathrm{mm}$ port placed in the same place as the median 11-mm port is placed for right-sided operation (Figure 3), because in the most cases it is not necessary to use 10 -mm tools for the left hand. To avoid an instrumental conflict, the distance between the ports has to be at least $5 \mathrm{~cm}$.

On the left side, the descending colon and the spleen have to be fully mobilized. Colon, spleen and pancreas are mobilizing medially until the adrenal gland is fully visualized. A special attention has to be paid to the pancreas tail, which in some cases can be mistaken for the adrenal gland. Leaning on the PLA experience gained in our clinic, we always try to achieve the maximal pancreas tale mobilization to be able to fully visualize the medial border of the adrenal gland. This technique helps to better assess the adrenal gland anatomy, the peculiarities of its blood supply and use these findings for a more effective planning of anatomical resection.

On the right side, a triangular ligament of the liver is dissected maximally cranially to ensure a liver mobility. The liver is retracted upward with the help of the liver retractor. Following liver retraction, it is possible to access to the right adrenal gland without colon mobilization. For better visualization of inferior vena cava, duodenum is mobilizing medially. To avoid a duodenum damage, the use of coagulation has to be minimized.

Following the tumor resection border assessment, adrenal gland tissue is clipped with big Hem-o-lok clips, leaving roughly $0.2-0.5 \mathrm{~cm}$ to the visible tumor border 

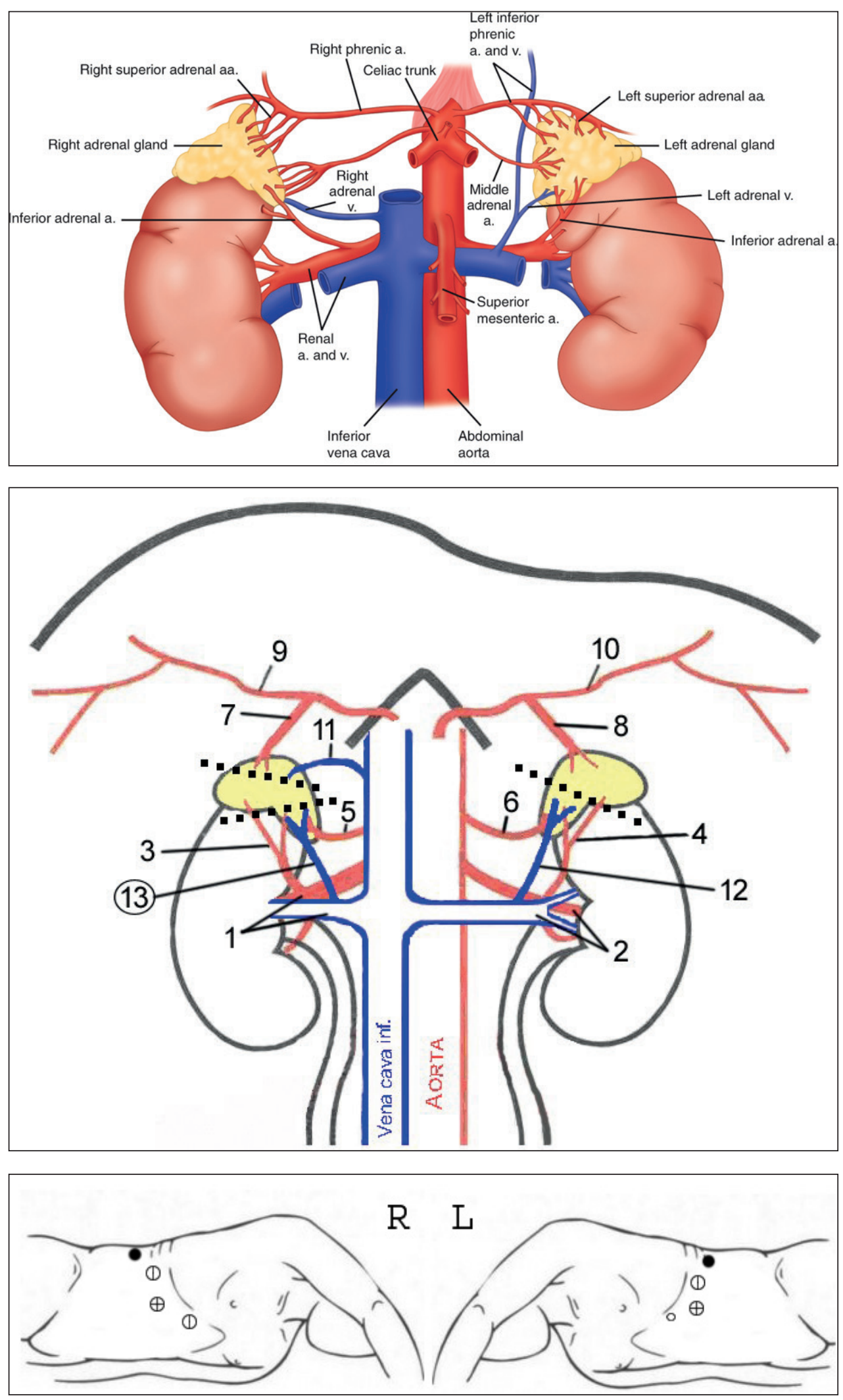

Fig. 1. Adrenal Gland Blood Supply

Fig. 2. Partial Adrenalectomy Options. (Signed resection areas/Dotted Lines $=$ Resection Areas). 1-Right renal artery and vena, 2- Left renal artery and vena, 3-Right inferior adrenal artery, 4- Left inferior adrenal artery, 5- Right middle adrenal artery, 6Left middle adrenal artery, 7- Right superior adrenal artery, 8- Left superior adrenal artery, 9-Right inferior phrenic artery, 10- Left inferior phrenic artery, 11-Right adrenal vena, 12- Left adrenal vena, 13-Accessory right adrenal vena ( $25 \%$ cases).

Fig. 3. Trocar placement: $\oplus$ - Optic (10mm), $\circledR$ - $11 \mathrm{~mm}$ trocar, 0 - $6 \mathrm{~mm}$ trocar, $\bullet$ - additional trocar. along the entire length (Figure 4). The safety margin has not yet been determined. Keeping in mind the fact that partial adrenalectomy is commonly indicated in the case of small benign tumors, we believe that under sufficient visualization of tumor border, "protective" margin of $2 \mathrm{~mm}$ of intact tissue is sufficient to prevent a false tumor relapse. Klaus J. [12] offers to reach hemostasis using bipolar coag- ulation and fibrin glue. The "cold" scissors resection after preliminary clipping makes it possible to better control the resection margin, as opposed to coagulation hemostasis. Preliminary clips-assisted tumor isolation also helps to prevent catecholamines ejection into the blood, which can cause a poorly treated hypertensive crisis. In relation to partial adrenalectomy, 

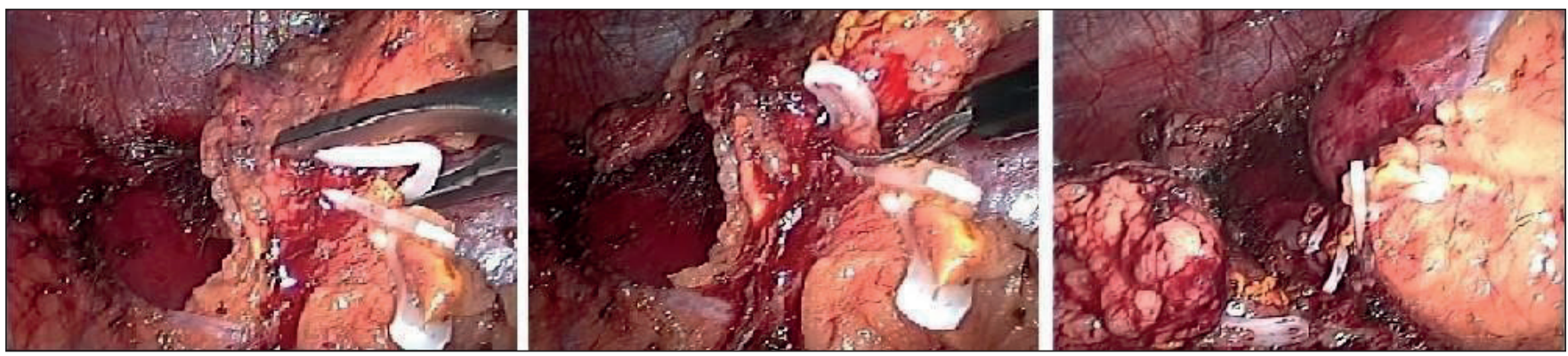

Fig. 4. Resection Margin Clipping with Hem-0-lok Clips.

Table I. Adrenal Gland Tumors: Histological Types.

\begin{tabular}{cccc}
\hline & PLA(n-14) & TLA (n-33) \\
\hline Right-sided & $6(43 \%)$ & $18(55 \%)$ \\
\hline Left-sided & $8(57 \%)$ & $15(45 \%)$ \\
\hline Median age (years) & 63 & 62 \\
\hline & Histological type & \\
\hline & Malignant tumors & & \\
\hline & & $4(14 \%)$ \\
\hline Primary adrenal gland tumor (adrenocortical cancer) & - & $4(14 \%)$ \\
\hline Lung cancer metastasis & - & $1(3 \%)$ \\
\hline Uterine carcinoma metastasis & - & \\
\hline & Benign tumors & $18(63 \%)$ \\
\hline Adenoma & $6(43 \%)$ & $6(21 \%)$ \\
\hline Pheochromocytoma & $2(14 \%)$ & - \\
\hline Cysts & $4(28 \%)$ & - \\
\hline Ganglioneuroma & $2(14 \%)$ & $19(58 \%)$ \\
\hline Hormone-producing tumors & $8(57 \%)$ & $14(42 \%)$ \\
\hline Non hormone-producing tumors & $6(43 \%)$ & \\
\hline
\end{tabular}

G. Cavallaro [13] offers to ligate preliminary adrenal vein, yet, our approach makes it possible to avoid ligation. The tumor is extracted from the wound with a container. Blake Drain $18 \mathrm{Fr}$ is applied to the resection area for 24 hours.

In the preoperative period, the patients with hormone-producing tumors received alpha-blockers for 2 weeks. Antibiotic prophylaxis was performed intraoperatively. In early postoperative period, thromboprophylaxis was performed according to the current international guidelines. Analgesic infusion was necessary only within the first 12 hours after the operation. Enteral feeding starts 10 hours after operation.

The histopathological examination of all operated patients (Table 1) detected 9 (19.1\%) malignant tumors, including 5 metastatic tumors (4 cases of lung tumor metastasis and 1 uterine carcinoma metastasis). Benign tumors includes 24 (63\%) adenomas, 8 (21\%)

pheochromocytomas, $4(10,5 \%)$ cysts and $2(5,5 \%)$ ganglioneuromas. Post-PLA

histopathological findings revealed 6 adenomas, 2 pheochromocytomas, 4 cysts and 2 ganglioneuromas. The median TLA time was $70 \mathrm{~min}$. (60-80 $\mathrm{min})$, PLA - 60 min. (50-70 min.). No significant operation time difference between TLA and PLA groups was detected. $(p>0,8)$. The median blood loss was $80 \mathrm{ml}(50-250 \mathrm{ml})$ in TLA and 50 $\mathrm{ml}(20-80 \mathrm{ml})$ in PLA $(\mathrm{p}>0,05)$.

One case of postoperative complication was a post-TLA bleeding (Clavien-Dindo III), which necessitated re-intervention and endoscopic hemostasis. The symptoms of hypercorticism (Clavien-Dindo II) were detected in one post-TLA patient, were managed conservative. Nothing like this was detected in any PLA case. Local recurrence was not observed during a follow up (4-53 month) in both groups.

In this paper, we described a standardized step-by-step technique of partial laparoscopic adrenalectomy, which can be performed effectively and safety.

As any other surgery, PLA requires a thorough patient selection, which is essential particularly for an experience gaining surgeon.

The profound knowledge of left-sided and right-sided PLA is the best tool to minimize intraoperative complications. On the left side, left renal vein, pancreas tale, kidney upper pole and m. psoas can significantly facilitate an operative visualization. As for the right side, it is necessary to visualize inferior vena cava, right renal vein, kidney upper pole and $\mathrm{m}$. psoas. Identification and visualization of blood vessels are the most difficult part of the adrenal 
gland surgery. Special attention has to be focused on the blood supply anatomy in the right-sided PLA.

\section{CONCLUSIONS}

Keeping in mind anatomical features of adrenal gland blood supply in highly-selected patients, PLA can be performed in a number of patients. $2 \mathrm{~mm}$ of a minimal resection margin is enough to preserve a false tumor recurrence. Anatomically grounded PLA is becoming a new standard of benign adrenal gland tumors treatment, providing an opportunity to save more adrenal gland functional tissue and to prevent hipocorticism development in postoperative period, which is particularly important for hereditary and bilateral tumors.

\section{REFERENCES}

1. Walz M.K., Peitgen K., Hoermann R.et al. Posterior retroperitoneoscopy as a new minimally invasive approach for adrenalectomy: results of 30 adrenalectomies in 27 patients, World J. Surg. 1996.20; (7):769-774.

2. Janetschek G., Lhotta K., Gasser R. et al. Adrenal-sparing laparoscopic surgery for aldosteroneproducing adenoma. J Endourol 1997;11:145-8.

3. Janetschek G., Finkenstedt G., Gasser R. et al. Laparoscopic surgery for pheochromocytoma: adrenalectomy, partial resection, excision of paragangliomas. J Urol 1998;160:330-4.

4. Neumann H.P.H., Reincke M., Bender B.U. et al. Preserved adrenocortical function after laparoscopic bilateral adrenal sparing surgery for hereditary pheochromocytoma. J Clin Endocrinol Metabol 1999; 84: 10.

5. Knežević N., Milas I., Kuliš T.et al. Partial laparoscopic adrenalectomy as a method of surgical management of adrenal tumors - Eur Urol Suppl 2017; 16(3): 563.

6. Nagaraja V., Guy D. et al. Recurrence and functional outcomes of partial adrenalectomy: A systematic review and meta-analysis - International Journal of Surgery 16 2015; (7):13.

7. Sapin M.R., Bilich G.L. Normal'naja anatomija cheloveka: Uchebnik. Medicinskoe informacionnoe agenstvo. 2010: 162 (in Russian).

8. Diner E.K., Franks M.E., Behari A. et al. Partial adrenalectomy: the National Cancer Institute experience. Urology. 2005; 66 (1): 19-23.

9. Styopushkin S., Chaikovskyi V., Sokolenko R. et al. Anatomical basis of laparoscopic partial adrenalectomy Eur Urol Suppl 2018; 17(2):34

10. N. Roukounakis, S. Dimas, I. Kafetzis, et al., Is preservation of the adrenal vein mandatory in laparoscopic adrenalsparing surgery? Jsls. 2007; 11(2): 215-218.

11. Hasson HM. A modified instrument and method for laparoscopy. Am J Obstet Gynecol 1971;110:886-7.

12. Jeschke K., Peschel R.. Zussner F. et al. Laparoscopic partial adrenalectomy for adenoma and familial pheochromocytoma. European Urology Supplements 12002; 1: 11.

13. Cavallaro G., Polistena A., D'Ermo G. et al. Partial adrenalectomy: when, where, and how? Considerations on technical aspect and indications to surgery. Eur Surg. 2012; 44(3): 150-154

\section{ORCID and contributionship:}

Sergiy P. Styopushkin: 0000-0001-7841-2484 ${ }^{\text {A, C, E, F }}$

Viktor P. Chaikovskyi: 0000-0001-7974-3656 ${ }^{A, B, C, D}$

Volodymyr A. Chernylovskyi: 0000-0002-5795-1574 A,B, C, D, E, F

Ruslan V. Sokolenko: 0000-0002-9083-4177 ${ }^{A, B}$

\section{Conflict of interest:}

The Authors declare no conflict of interest.

\section{CORRESPONDING AUTHOR Volodymyr A. Chernylovskyi \\ City Clinical Hospital №4 \\ 31 Blizhnaya st., 49000 Dnipro, Ukraine \\ tel: +380973695672 \\ e-mail: chernylovskyi@gmail.com}

Received: 12.11.2019

Accepted: 26.06 .2020

A - Work concept and design, B - Data collection and analysis, C - Responsibility for statistical analysis, D-Writing the article, $\mathbf{E}$-Critical review, $\mathbf{F}$ - Final approval of the article 